\title{
CLT for single functional index quantile regression under dependence structure
}

\author{
Nadia Kadiri \\ University Djillali LIABES of Sidi Bel \\ Abbes, Algeria \\ email: nad.kad06@yahoo.com \\ Salah Khardani \\ Laboratoire de Physique Mathématique, \\ Fonctions spéciales et Applications, \\ Hammam-Sousse, Tunisia \\ email: khardani_salah@yahoo.fr
}

\author{
Abbes Rabhi \\ University Djillali Liabes of Sidi Bel \\ Abbes, Algeria \\ email: rabhi_abbes@yahoo.fr \\ Fatima Akkal \\ University Djillali LIABES of Sidi Bel \\ Abbes, Algeria \\ email: fatima.akkal@hotmail.com
}

\begin{abstract}
In this paper, we investigate the asymptotic properties of a nonparametric conditional quantile estimation in the single functional index model for dependent functional data and censored at random responses are observed. First of all, we establish asymptotic properties for a conditional distribution estimator from which we derive an central limit theorem (CLT) of the conditional quantile estimator. Simulation study is also presented to illustrate the validity and finite sample performance of the considered estimator. Finally, the estimation of the functional index via the pseudo-maximum likelihood method is discussed, but not tackled.
\end{abstract}

\section{Introduction}

Multivariate regression analysis is a powerful statistical tool in biomedical research and many fields of life (Muharisa et al. [27]) with numerous applications.

2010 Mathematics Subject Classification: Primary 62G05, Secondary 62G99, Thirdly $62 \mathrm{M} 10$

Key words and phrases: conditional quantile, censored data, functional random variable, Kernel estimator, nonparametric estimation, probabilities of small balls, strong mixing processes, single index model 
While linear regression can be used to model the expected value (ie, mean) of a continuous outcome given the covariates in the model, quantile regression can be used to compare the entire distribution of a continuous response or a specific quantile of the response between groups. Despite the regression function is of interest, other statistics such as quantile and mode regression might be important from a theoretical and a practical point of view. Quantile regression is a common way to describe the dependence structure between a response variable $\mathrm{Y}$ and some covariate $\mathrm{X}$. Unlike the regression function that relies only on the central tendency of the data, the conditional quantile function allows the analyst to estimate the functional dependence between variables for all portions of the conditional distribution of the response variable. Moreover, it is well known that conditional quantiles can give a good description of the data (see, Chaudhuri et al. [9]), such as robustness to heavy-tailed error distributions and outliers to ordinary mean-based regression. As a particular case, note that the conditional median is useful for asymmetric distributions.

Quantile regression(QR) is one of the major statistical tools and is gradually developing into a comprehensive strategy for completing the regression prediction. It is emerging as a popular statistical approach, which complements the estimation of conditional mean models. While the latter only focuses on one aspect of the conditional distribution of the dependent variable, the mean, quantile regression provides more detailed insights by modeling conditional quantiles. Her can therefore detect whether the partial effect of a regressor on the conditional quantiles is the same for all quantiles or differs across quantiles, and can provide evidence for a statistical relationship between two variables even if the mean regression model does not. In many fields of applications like quantitative finance, econometrics, marketing and also in medical and biological sciences, QR is a fundamental element for data analysis, modeling and inference. An application in finance is the analysis of conditional Valueat-Risk, moreover, her is the development of statistical tools used to explain the relationship between response and predictor variables (see Yanuar et al. [37]). The quantile method is a technique of dividing a group of data into several parts after the data is sorted from the smallest to the largest Yanuar et al. [36]. QR enjoys some very appealing features. Apart from enabling some very exible patterns of partial effects, quantile regressions are also interesting because they satisfy some equivariance and robustness principles.

The advantage of the QR methodology is that it allows for understanding relationships between variables outside of the conditional mean of the response; it is useful for understanding an outcome at its various quantiles and comparing groups or levels of an exposure on those quantiles. QR is a common way 
to describe the dependence structure between a response variable $Y$ and some covariate $X$. Unlike the regression function (which is defined as the conditional mean) that relies only on the central tendency of the data, the conditional quantile function allows the analysts to estimate the functional dependence between variables for all portions of the conditional distribution of the response variable. Moreover, quantiles are well known for their robustness to heavy-tailed error distributions and outliers which allow to consider them as a useful alternative to the regression function Chaouch and Khardani [8].

Moreover, it is a statistical technique intended to estimate, and conduct inference about, conditional quantile functions. Just as classical linear regression methods based on minimizing sums of squared residuals enable one to estimate models for conditional mean functions, quantile regression methods offer a mechanism for estimating models for the conditional median function, and the full range of other conditional quantile functions. By supplementing the estimation of conditional mean functions with techniques for estimating an entire family of conditional quantile functions, quantile regression is capable of providing a more complete statistical analysis of the stochastic relationships among random variables.

For example, QR has been used in a broad range of application settings. Reference growth curves for children's height and weight have a long history in pediatric medicine; quantile regression methods may be used to estimate upper and lower quantile reference curves as a function of age, sex, and other covariates without imposing stringent parametric assumptions on the relationships among these curves. In ecology, theory often suggests how observable covariates affect limiting sustainable population sizes, and quantile regression has been used to directly estimate models for upper quantiles of the conditional distribution rather than inferring such relationships from models based on conditional central tendency. In survival analysis, and event history analysis more generally, there is often also a desire to focus attention on particular segments of the conditional distribution, for example survival prospects of the oldest-old, without the imposition of global distributional assumptions.

In recent years, estimating conditional quantiles has received increasing interest in the literature, for both independent and dependent data; Samanta [31] established a nonparametric estimation of conditional quantiles, Wang and Zhao [35] presented a kernel estimator for conditional t-quantiles for mixing samples and established its strong uniform convergence. Ferraty et al. [15] studied the estimation of a conditional quantiles for functional dependent data with application to the climatic El Ninö phenomenon. Ezzahrioui \& Elias Ould-Saïd [14] considered the estimation of the conditional quantile function 
when the covariates take values in some abstract function space, the almost complete convergence and the asymptotic normality of the kernel estimator of the conditional quantile under the $\alpha$-mixing assumption were established. Ferraty et al. [15] introduced a nonparametric estimator of the conditional quantile defined as the inverse of the conditional cumulative distribution function (df) when data are dependent.

In life time data analysis, nonparametrically estimated conditional survival curves (such as the conditional Kaplan-Meier estimate) are useful for assessing the influence of risk factors, predicting survival probabilities, and checking goodness-of-fit of various survival regression models. It is well known that in medical studies the observation on the survival time of a patient is often incomplete due to right censoring. Classical examples of the causes of this type of censoring are that the patient was alive at the termination of the study, that the patient withdrew alive during the study, or that the patient died from other causes than those under study. The censored quantile regression model is derived from the censored model. This method is used to overcome problems in modeling censored data as well as to overcome the assumptions of linear models that are not met, in this linear models Sarmada and Yanuar [32] have compared the results of the analysis of the quantile regression method with the censored quantile regression method for censored data. In the context of censored data, Gannoun et al. [17] introduced a local linear (LL) estimator of the quantile regression and established its almost sure consistency (without rate) as well as its asymptotic normality in the independent and identically distributed (i.i.d.) case. El Ghouch and Van Keilegom [13] considered the LL estimation of the quantile regression and its first derivative under an $\alpha$-mixing assumption and studied their asymptotic properties. Ould-Saïd [28] constructed a kernel estimator of the conditional quantile under an i.i.d. censorship model and established its strong uniform convergence rate. Under an $\alpha$-mixing assumption, Liang and Alvarez [21] established the strong uniform convergence (with rate) of the conditional quantile function as well as its asymptotic distribution.

The single index model is a natural extension of the linear regression model for applications in which linearity does not hold. This last approach is widely applied in econometrics as a reasonable compromise between nonparametric and parametric models. In the past few recent years, the single functional index models have received much attention, and it has been studied extensively in both statistical and econometric literatures. Interesting to this methods, many authors worked on this sort of problems, see for instance Aït-Saidi et al. [1,2]. Attaoui et al. [3] investigated the kernel estimator of the conditional density 
of a scalar response variable $Y$, given a Hilbertian random variable $X$ when the observations are from a single functional index model. Ling et al. [24] reconsidered the kernel estimator of the conditional density when the scalar response variable $Y$ and the Hilbertian random variable $X$ also come from the single functional index model. The asymptotic results such as pointwise almost complete consistency and the uniform almost complete convergence of the kernel estimation with rates in the setting of the $\alpha$ mixing functional data are also obtained, which extend the i.i.d. case in Attaoui et al. [3] to the dependence setting. Ling \& Xu [23] investigated the estimation of conditional density function based on the single-index model for functional time series data. Under $\alpha$-mixing condition, the asymptotic normality of the conditional density estimator and the conditional mode estimator where obtained. Attaoui [4] studied a nonparametric estimation of the conditional density of a scalar response variable given a random variable taking values in separable Hilbert space when the variables satisfy the strong mixing dependency, based on the single-index structure.

Inspired by all the papers above, our work in this paper aims to contribute to the research on functional nonparametric regression model, by giving an alternative estimation of $\mathrm{QR}$ estimation in the single functional index model with randomly right-censored data under $\alpha$-mixing conditions whose definition is given below.

Recall that a process $\left(X_{i}, Y_{i}\right)_{i \geq 1}$ is called $\alpha$-mixing or strongly mixing (see Lin and $\mathrm{Lu} \mathrm{[22])} \mathrm{for} \mathrm{more} \mathrm{details} \mathrm{and} \mathrm{examples,} \mathrm{if}$

$$
\sup _{k} \sup _{A \in \mathcal{F}_{1}^{k}} \sup _{B \in \mathcal{F}_{n+k}^{\infty}}|\mathbb{P}(A \cap B)-\mathbb{P}(A) \mathbb{P}(B)|=\alpha(n) \rightarrow 0 \text { as } n \rightarrow \infty \text {, }
$$

where $\mathcal{F}_{j}^{k}$ denotes the $\sigma$-field generated by the random variables $\left\{\left(X_{i}, Y_{i}\right), j \leq\right.$ $i \leq k\}$. The process $\left\{\left(X_{i}, Y_{i}\right), i \geq 1\right\}$ is said to be arithmetically $\alpha$ mixing with order $a>0$, if $\exists C>0, \alpha(n) \leq C n^{-a}$.

The strong-mixing condition is reasonably weak and has many practical applications (see, e.g., Cai [6], Doukhan [11], Dedecker et al. [10] Ch. 1, for more details). In particular, Masry and Tøjstheim [25] proved that, both ARCH processes and nonlinear additive autoregressive models with exogenous variables, which are particularly popular in finance and econometrics, are stationary and $\alpha$-mixing.

This article is organized as follows: In Section 2, we describe our model and construct precisely the QR estimator based on the functional stationary data under censorship model. In Section 3, we build up asymptotic theorems for our model. Section 4 illustrates those asymptotic properties through some 
simulated. Finally, the proofs of the main results are postponed to Section 5 .

\section{Notations and estimators of the semi-parametric framework}

\subsection{The model}

Let $(X, T)$ be a pair of random variables where $T$ is a real-valued random variable and $X$ takes its values in a separable Hilbert space $\mathcal{H}$ with the norm $\|\cdot\|$ generated by an inner product $\langle\cdot, \cdot\rangle$. Let $C$ be a censoring variable with common continuous distribution function $\mathrm{G}$. The continuity of $\mathrm{G}$ allows to use the convergence results for the Kaplan and Meier estimator of G. (see [19]).

From now on we suppose that $(X, T)$ and $C$ are independent. It is plausible whenever the censoring is independent of the characteristics of the patients under study. In the right censorship model, the pair $(T, C)$ is not directly observed and the corresponding available information is given by $\mathrm{Y}=\min (\mathrm{T}, \mathrm{C})$ and $\delta=\mathbf{1}_{\{\mathrm{T} \leq \mathrm{C}\}}$, where $\mathbf{1}_{\mathrm{A}}$ is the indicator function of the set $A$.

Such censorship models have been amply studied in the Literature for real or multi-dimensional random variables, and in nonparametric frameworks the kernel techniques are particularly used (see Tanner and Wong [33], Padgett [29], Lecoutre and Ould-Saïd [20] and Van Keilegom and Veraverbeke [34], for a necessarily non-exhaustive sample of literature in this area).

Furthermore, let $\left(X_{i}, T_{i}\right)_{1 \leq i \leq n}$ be the statistical sample of pairs which are identically distributed like $(X, T)$, but not necessarily independent, $\left(C_{i}\right)_{1 \leq i \leq n}$ is a sequence of i.i.d. random variables which is independent of $\left(X_{i}, T_{i}\right)_{1 \leq i \leq n}$. Therefore, we assume that the sample $\left\{\left(X_{i}, \delta_{i}, Y_{i}\right), i=1, \ldots, n\right\}$ is at our disposal. Moreover, we consider $d_{\theta}(\cdot, \cdot)$ a semi-metric associated with the single index $\theta \in \mathcal{H}$ defined by $d_{\theta}\left(x_{1}, x_{2}\right):=\left|<x_{1}-x_{2}, \theta\right\rangle \mid$, for $x_{1}$ and $x_{2}$ in $\mathcal{H}$.

For a fixed $x$ in $\mathcal{H}$, the conditional cumulative distribution function (cond$c d f$ ) of $Y$ given $\langle\theta, X\rangle=\langle\theta, x\rangle$, is defined as follows:

$$
\forall \mathrm{t} \in \mathbb{R}, \mathrm{F}(\theta, \mathrm{t}, x):=\mathbb{P}(\mathrm{Y} \leq \mathrm{t} \mid<\mathrm{X}, \theta>=<x, \theta>) .
$$

Saying that, we are implicitly assuming the existence of a regular version for the conditional distribution of $Y$ given $\langle\theta, X\rangle$. Now, let $\zeta_{\theta}(\gamma, x)$ be the $\gamma$ th-conditional quantile of the distribution of $Y$ given $\langle\theta, X\rangle=\langle\theta, x\rangle$. Formally, $\zeta_{\theta}(\gamma, x)$ is defined as:

$$
\zeta_{\theta}(\gamma, x):=\inf \{t \in \mathbb{R}: F(\theta, t, x) \geq \gamma\}, \quad \forall \gamma \in(0,1) .
$$


In order to simplify our framework and to focus on the main interest of our paper (the functional feature of $\langle\theta, X\rangle$ ), we assume that $F(\theta, \cdot, x)$ is strictly increasing and continuous in a neighborhood of $\zeta_{\theta}(\gamma, x)$. This is insuring that the conditional quantile $\zeta_{\theta}(\gamma, x)$ is uniquely defined by:

$$
\zeta_{\theta}(\gamma, x)=F^{-1}(\theta, \gamma, x) \text { equivalently } \widehat{F}\left(\theta, \widehat{\zeta}_{\theta}(\gamma, x), x\right)=\gamma \text {. }
$$

Next, in all what follows, we assume only smoothness restrictions for the cond-cdf $\mathrm{F}(\theta, \cdot, x)$ through nonparametric modeling. Assume also $\left(\mathrm{X}_{i}, \mathrm{~T}_{i}\right)_{i \in \mathbb{N}}$ is an $\alpha$-mixing sequence, which is one among the most general mixing structures.

\subsection{The estimators}

The kernel estimator $F_{n}(\theta, \cdot, x)$ of $F(\theta, \cdot, x)$ is presented as follows:

$$
F_{n}(\theta, t, x)=\frac{\sum_{i=1}^{n} K\left(h_{K}^{-1}\left(<x-X_{i}, \theta>\right)\right) H\left(h_{H}^{-1}\left(t-T_{i}\right)\right)}{\sum_{i=1}^{n} K\left(h_{K}^{-1}\left(<x-X_{i}, \theta>\right)\right)},
$$

where $\mathrm{K}$ is a kernel function, $\mathrm{H}$ a cumulative distribution function and $h_{K}=$ $h_{K, n}$ (resp. $h_{H}=h_{H, n}$ ) a sequence of positive real numbers. Note that using similar ideas, Roussas [30] introduced some related estimates but in the special case when $X$ is real, while Samanta [31] produced previous asymptotic study.

As a by-product of (1) and (2), it is easy to derive an estimator $\zeta_{\theta, n}(\gamma, x)$ of $\zeta_{\theta}(\gamma, x)$ :

$$
\zeta_{\theta, n}(\gamma, x)=F_{n}^{-1}(\theta, \gamma, x) .
$$

Such an estimator is unique as soon as $\mathrm{H}$ is an increasing continuous function. Such an approach has been largely used in the case where the variable $X$ is of finite dimension (see e.g Whang and Zhao [35], Cai [7], Zhou and Liang [38] or Gannoun et al. [17]).

The objective of this section is to adapt these ideas under functional random variable $X$, and build a kernel type estimator of the conditional distribution $F(\theta, \cdot, X)$ adapted for censored samples. In the censoring case, based on the observed sample $\left(X_{i}, \delta_{i}, Y_{i}\right)_{i=1, \ldots, n}$ we define the following "pseudo-estimator" of $F(\theta, \cdot, X)$ which is used as an intermediate estimator, thus we can reformulate 
the expression (2) as follows:

$$
\widetilde{\mathrm{F}}(\theta, t, x)=\frac{\sum_{i=1}^{n} \frac{\delta_{i}}{\bar{G}\left(Y_{i}\right)} K\left(h_{K}^{-1}\left(<x-X_{i}, \theta>\right)\right) H\left(h_{H}^{-1}\left(t-Y_{i}\right)\right)}{\sum_{i=1}^{n} K\left(h_{K}^{-1}\left(<x-X_{i}, \theta>\right)\right)} .
$$

In practice $\overline{\mathrm{G}}(\cdot)=1-\mathrm{G}(\cdot)$ is unknown, hence it is impossible to use the estimator (6). Then, we replace $\overline{\mathrm{G}}(\cdot)$ by its Kaplan and Meier [19] estimate $\overline{\mathrm{G}}_{\mathfrak{n}}(\cdot)$ given by

$$
\bar{G}_{n}(t)=1-G_{n}(t)= \begin{cases}\prod_{i=1}^{n}\left(1-\frac{1-\delta_{(i)}}{n-i+1}\right)^{\mathbf{1}_{\left\{Y_{(i)} \leq t\right\}},}, & \text { if } t<Y_{(n)} \\ 0, & \text { if } t \geq Y_{(n)} .\end{cases}
$$

where $Y_{(1)}<Y_{(2)}<\ldots<Y_{(n)}$ are the order statistics of $Y_{i}$ and $\delta_{(i)}$ is the concomitant of $Y_{(i)}$. Therefore, a full estimator of the conditional distribution function $F(\theta, \cdot, x)$ is defined as:

$$
\widehat{F}(\theta, t, x)=\frac{\sum_{i=1}^{n} \frac{\delta_{i}}{\bar{G}_{n}\left(Y_{i}\right)} K\left(h_{K}^{-1}\left(<x-X_{i}, \theta>\right)\right) H\left(h_{H}^{-1}\left(t-Y_{i}\right)\right)}{\sum_{i=1}^{n} K\left(h_{K}^{-1}\left(<x-X_{i}, \theta>\right)\right)},
$$

which is rewritten also as:

$$
\widehat{F}(\theta, t, x)=\frac{\widehat{F}_{N}(\theta, t, x)}{\widehat{F}_{D}(\theta, x)} .
$$

Consequently, a natural estimator of $\zeta_{\theta}(\gamma, x)$ is given by

$$
\begin{aligned}
\widehat{\zeta}_{\theta}(\gamma, x) & =\widehat{F}^{-1}(\theta, \gamma, x) \\
& =\inf \{t \in \mathbb{R}: \widehat{F}(\theta, t, x) \geq \gamma\},
\end{aligned}
$$

which satisfies

$$
\widehat{\mathrm{F}}\left(\theta, \widehat{\zeta}_{\theta}(\gamma, x), x\right)=\gamma
$$




\section{Assumptions and results}

\subsection{Assumptions on the functional variable}

Let $N_{x}$ be a fixed neighborhood of $x$ and let $B(x, h)$ be the ball of center $x$ and radius $h$, namely $B_{\theta}(x, h)=\{f \in \mathcal{H} / 0<|<x-f, \theta>|<h\}$. Assume that, $\left(C_{i}\right)_{i \geq 1}$ and $\left(T_{i}\right)_{i \geq 1}$ are independent and we assume that $\tau_{G}:=\sup \{t$ : $\mathrm{G}(\mathrm{t})<1\}$ and let $\tau$ be a positive real number such that $\tau<\tau_{\mathrm{G}}$.

let's consider the following hypotheses:

(H1) $\forall \mathrm{h}>0, \mathbb{P}\left(\mathrm{X} \in \mathrm{B}_{\theta}(\mathrm{x}, \mathrm{h})\right)=\phi_{\theta, \mathrm{x}}(\mathrm{h})>0$,

(H2) $\left(X_{i}, Y_{i}\right)_{i \in \mathbb{N}}$ is an $\alpha$-mixing sequence whose the coefficients of mixture verify:

$$
\exists \mathrm{a}>0, \exists \mathrm{c}>0: \forall \mathrm{n} \in \mathbb{N}, \alpha(\mathrm{n}) \leq \mathrm{cn}^{-\mathrm{a}} .
$$

(H3) $0<\sup _{i \neq j} \mathbb{P}\left(\left(X_{i}, X_{j}\right) \in B_{\theta}(x, h) \times B_{\theta}(x, h)\right)=\mathcal{O}\left(\frac{\left(\phi_{\theta, x}\left(h_{K}\right)\right)^{(a+1) / a}}{n^{1 / a}}\right)$.

\section{$3.2 \quad$ The nonparametric model}

As usually in nonparametric estimation, we suppose that the cond-cdf $F(\theta, \cdot, \chi)$ verifies some smoothness constraints. Let $b_{1}$ and $b_{2}$ be two positive numbers; such that:

(H4) $\forall\left(x_{1}, x_{2}\right) \in \mathrm{N}_{\mathrm{x}} \times \mathrm{N}_{\mathrm{x}}, \forall\left(\mathrm{t}_{1}, \mathrm{t}_{2}\right) \in \mathcal{S}_{\mathbb{R}}^{2}$,

(i) $\left|F\left(\theta, t_{1}, x_{1}\right)-F\left(\theta, t_{2}, x_{2}\right)\right| \leq C_{\theta, x}\left(\left\|x_{1}-x_{2}\right\|^{b_{1}}+\left|t_{1}-t_{2}\right|^{b_{2}}\right)$,

(ii) $\int_{\mathbb{R}} \mathrm{tf}(\theta, \mathrm{t}, x) \mathrm{dt}<\infty$ for all $\theta, x \in \mathcal{H}$.

To this end, we need some assumptions concerning the kernel estimator $\widehat{\mathrm{F}}(\theta, \cdot, x)$ :

(H5) $\forall\left(t_{1}, t_{2}\right) \in \mathbb{R}^{2},\left|H\left(t_{1}\right)-H\left(t_{2}\right)\right| \leq C\left|t_{1}-t_{2}\right|$ with $\int H^{(1)}(t) d t=1$, $\int H^{2}(t) d t<\infty$ and $\int|t|^{b_{2}} H^{(1)}(t) d t<\infty$.

(H6) $\mathrm{K}$ is a positive bounded function with support $[0,1]$.

(H7) The df of the censored random variable, $\mathrm{G}$ has bounded first derivative $\mathrm{G}^{\prime}$. 
(H8) For all $u \in[0,1], \lim _{h \rightarrow 0} \frac{\phi_{\theta, x}(u h)}{\phi_{\theta, x}(h)}=\lim _{h \rightarrow 0} \xi_{h}^{\theta, x}(u)=\xi_{0}^{\theta, x}(u)$.

(H9) The bandwidth $h_{H}$ satisfies,

(i) $\mathrm{nh}_{\mathrm{H}}^{2} \phi_{\theta, x}^{2}\left(\mathrm{~h}_{\mathrm{K}}\right) \longrightarrow \infty$, and $\frac{\mathrm{nh}_{\mathrm{H}}^{3} \phi_{\theta, \mathrm{x}}\left(\mathrm{h}_{\mathrm{K}}\right)}{\log ^{2} \mathrm{n}} \longrightarrow \infty$ as $\mathrm{n} \rightarrow \infty$.

(ii) $\mathrm{nh}_{\mathrm{H}}^{2} \phi_{\theta, x}^{3}\left(\mathrm{~h}_{\mathrm{K}}\right) \longrightarrow 0$, as $\mathrm{n} \rightarrow \infty$.

(H10) There exist sequences of integers $\left(u_{n}\right)$ and $\left(v_{n}\right)$ increasing to infinity such that $\left(u_{n}+v_{n}\right) \leq n$, satisfying

(i) $v_{n}=o\left(\left(n \phi_{\theta, x}\left(h_{K}\right)\right)^{1 / 2}\right)$ and $\left(\frac{n}{\phi_{\theta, x}\left(h_{K}\right)}\right)^{1 / 2} \alpha\left(v_{n}\right) \rightarrow 0$ as $n \rightarrow 0$,

(ii) $\mathrm{q}_{\mathrm{n}} v_{\mathrm{n}}=\mathrm{o}\left(\left(\mathrm{n} \phi_{\theta, \mathrm{x}}\left(\mathrm{h}_{\mathrm{K}}\right)\right)^{1 / 2}\right)$ and $\mathrm{q}_{\mathrm{n}}\left(\frac{\mathrm{n}}{\phi_{\theta, \mathrm{x}}\left(\mathrm{h}_{\mathrm{K}}\right)}\right)^{1 / 2} \alpha\left(v_{\mathrm{n}}\right) \rightarrow 0$ as $\mathrm{n} \rightarrow 0$

where $q_{n}$ is the largest integer such that $q_{n}\left(u_{n}+v_{n}\right) \leq n$.

\subsection{Comments of the assumptions}

(H1) can be interpreted as a concentration hypothesis acting on the distribution of the f.r.v. $\mathrm{X}$, while (H3) concerns the behavior of the joint distribution of the pairs $\left(X_{i}, X_{j}\right)$. Indeed, this hypothesis is equivalent to assume that, for $n$ large enough

$$
\sup _{i \neq j} \frac{\mathbb{P}\left(\left(X_{i}, X_{j}\right) \in B_{\theta}(x, h) \times B_{\theta}(x, h)\right)}{\mathbb{P}\left(X \in B_{\theta}(x, h)\right)} \leq C\left(\frac{\phi_{\theta, x}\left(h_{K}\right)}{n}\right)^{1 / a} .
$$

This is one way to control the local asymptotic ratio between the joint distribution and its margin. Remark that the upper bound increases with a. In other words, more the dependence is strong, more restrictive is (H3). The hypothesis (H2) specifies the asymptotic behavior of the $\alpha$-mixing coefficients. Let's note that (H4) is used for the prove of the the almost complete convergence of $\widehat{\zeta}_{\theta}(\gamma, x)$. Assumptions (H5), (H6) and (H7) are classical in nonparametric estimation. To establish the asymptotic normality, dealing with strong mixing random variables (under (H2)), we use the well-known sectioning device introduced by Doob [12] in (H10).

This part of paper is devoted to the main result, the asymptotic normality of $\widehat{F}(\theta, t, x)$ and $\widehat{\zeta}_{\theta}(\gamma, x)$. 
Theorem 1 Under Assumptions (H1)-(H10), we have

$$
\left(\frac{n \phi_{\theta, x}\left(h_{K}\right)}{\sigma^{2}(\theta, t, x)}\right)^{1 / 2}(\widehat{F}(\theta, t, x)-F(\theta, t, x)) \stackrel{\mathcal{D}}{\longrightarrow} \mathcal{N}(0,1),
$$

where $\sigma^{2}(\theta, t, x)=\frac{a_{2}(\theta, x)}{\left(a_{1}(\theta, x)\right)^{2}} F(\theta, t, x)\left(\frac{1}{\bar{G}(t}-F(\theta, t, x)\right)$

and

$$
\frac{\phi_{\theta, x}\left(h_{K}\right) \mathbb{E} K_{1}^{2}(x, \theta)}{\mathbb{E}^{2} K_{1}(x, \theta)}=: \frac{a_{2}(\theta, x)}{\left(a_{1}(\theta, x)\right)^{2}} .
$$

Theorem 2 If the Assumptions (H1)-(H10) are satisfied, and et $\gamma$ is the unique order of the quantile such that $\gamma=\mathrm{F}\left(\theta, \zeta_{\theta}(\gamma, x), x\right)=\mathrm{F}_{\mathrm{n}}\left(\theta, \widehat{\zeta}_{\theta}(\gamma, x), x\right)$,

$$
\left(\frac{n \phi_{\theta, x}\left(h_{\mathrm{K}}\right)}{\Sigma^{2}\left(\theta, \zeta_{\theta}(\gamma, x), x\right)}\right)^{1 / 2}\left(\zeta_{\theta, n}(\gamma, x)-\zeta_{\theta}(\gamma, x)\right) \stackrel{\mathcal{D}}{\longrightarrow} \mathcal{N}(0,1)
$$

where $\Sigma\left(\theta, \zeta_{\theta}(\gamma, x), x\right)=\frac{\sigma\left(\theta, \zeta_{\theta}(\gamma, x), x\right)}{f\left(\theta, \zeta_{\theta}(\gamma, x), x\right)}$.

As one can see, the asymptotic variance $\Sigma\left(\theta, \zeta_{\theta}(\gamma, x), x\right)$ depends on some unknown functions $f\left(\theta, \zeta_{\theta}(\gamma, x), x\right)$ and $\phi_{\theta, x}\left(h_{K}\right)$ and other theoretical quantities $F\left(\theta, \zeta_{\theta}(\gamma, x), x\right), \bar{G}(\cdot)$ and $\zeta_{\theta}(\gamma, x)$ that have to be estimated in practice. Therefore, $\bar{G}(\cdot), F(\theta, t, x)$ and $\zeta_{\theta}(\gamma, x)$ should be replaced, respectively, by the Kaplan-Meier's estimator $\bar{G}_{n}(\cdot)$, the kernel-type estimator of the joint distribution $\widehat{f}\left(\theta, \zeta_{\theta}(\gamma, x), x\right)$ and $\zeta_{\theta, n}(\gamma, x)$ the conditional quantile estimator given by equation (8). Moreover, using the decomposition given by assumption (H1), one can estimate $\phi_{\theta, x}(z)$ by $F_{x, n}(z)=1 / n \sum_{i=1}^{n} 1_{\left\{X_{i} \in B_{\theta}(x, z)\right\}}$.

The corollary below allows one to obtain a confidence interval in practice since all quantities are known.

\subsection{Confidence intervals}

Now based on the quantities estimation, we easily get a plug-in estimator $\widehat{\Sigma}\left(\theta, \zeta_{\theta, n}(\gamma, x), x\right)$ of $\Sigma\left(\theta, \zeta_{\theta}(\gamma, x), x\right)$. The Theorem (2) can be now used to provide the $100(1-\gamma) \%$ confidence bands for $\zeta_{\theta}(\gamma, x)$ which is given, for $x \in \mathcal{H}$, by

$$
\left[\zeta_{\theta, n}(\gamma, x)-c_{\gamma / 2} \frac{\hat{\Sigma}\left(\theta, \zeta_{\theta, n}(\gamma, x), x\right)}{\sqrt{n F_{x, n}\left(h_{K}\right)}}, \zeta_{\theta, n}(\gamma, x)+c_{\gamma / 2} \frac{\hat{\Sigma}\left(\theta, \zeta_{\theta, n}(\gamma, x), x\right)}{\sqrt{n F_{x, n}\left(h_{K}\right)}}\right]
$$

where $c_{\gamma / 2}$ is the upper $\gamma / 2$ quantile of the distribution of $\mathcal{N}(0,1)$. 


\section{Finite sample performance}

This section considers simulated as well as real data studies to assess the finitesample performance of the proposed estimator and compare it to its competitor. More precisely, we are interested in comparing the conditional quantile estimator based on single functional index model (SFIM) to the kernel-type conditional quantile estimator (NP) introduced in Chaouch and Khardani [8] when the data is dependent and the response variable is subject to a random right-censorship phenomena. Throughout the simulation part, the $\mathrm{n}$ i.i.d. random variables $\left(C_{i}\right)_{i}$ ( censured variables) are simulated through the exponential distribution $\mathcal{E}(1.5)$. Similarly, in the real data applications, the censored variables are simulated according to the aforementioned exponential law.

The single functional index $\theta \in \mathcal{H}$ is usually unknown and has to be estimated in practice. This topic was discussed in single functional regression model literature and an estimation approaches based on cross-validation or maximum-likelihood methods were discussed, for instance, in Aït Saidi et al. [2] and the references therein. Another alternative, which will be adopted in this section, consists in selecting $\theta(t)$ among the eigenfunctions of the covariance operator $\mathbb{E}\left[\left(X^{\prime}-\mathbb{E}\left(X^{\prime}\right)\right)<X^{\prime}, .>_{\mathcal{H}}\right]$, where $X(t)$ is, for instance, a diffusiontype process defined on a real interval $[a, b]$ and $X^{\prime}(t)$ its first derivative (see, for instance, Attaoui and Ling [5]). Given a training sample $\mathcal{L}$, the covariance operator can be estimated by its empirical version $\frac{1}{|\mathcal{L}|} \sum_{i \in \mathcal{L}}\left(X_{i}^{\prime}-\mathbb{E} X^{\prime}\right)^{\mathfrak{t}}\left(X_{i}^{\prime}-\right.$ $\left.\mathbb{E} X^{\prime}\right)$. Consequently, one can obtain a discretized version of the eigenfunctions $\theta_{i}(t)$ by applying the principle component analysis method. Let $\theta^{\star}$ be the first eigenfunction corresponding to the highest eigenvalue of the empirical covariance operator, which will replace $\theta$ in the simulation steps to calculate the estimator of the conditional distribution as well as the conditional quantiles.

\subsection{Simulation study}

We generate $n$ copies, say $\left(X_{i}, \delta_{i}, Y_{i}\right)_{i=1, \ldots, n}$, of $(X, \delta, Y)$, where $X$ and $Y$ are simulated according to the following functional regression model.

$$
\mathrm{T}_{i}=\mathrm{R}\left(\mathrm{X}_{\mathrm{i}}\right)+\epsilon_{i}, i=1, \ldots, \mathrm{n},
$$

where $\epsilon_{i}$ is the error assumed to be generated according to an autoregressive model defined as:

$$
\epsilon_{i}=1 / \sqrt{2} \epsilon_{i-1}+\eta_{i}, i=1, \ldots, n,
$$

where $\left(\eta_{i}\right)_{i}$ a sequence of i.i.d. random variables normally distributed with a variance equal to 0.1 . The functional covariate $X$ is assumed to be a diffusion 
process defined on $[0,1]$ and generated by the following equation:

$$
X(t)=A(2-\cos (\pi t W))+(1-A) \cos (\pi t W), t \in[0,1]
$$

where $W \rightsquigarrow \mathcal{N}(0,1)$ and $A \rightsquigarrow \operatorname{Bernoulli}(1 / 2)$.

Figure 1 depicts a sample of 100 realizations of the functional random variable $X$ sampled in 100 equidistant points over the interval $[0,1]$.

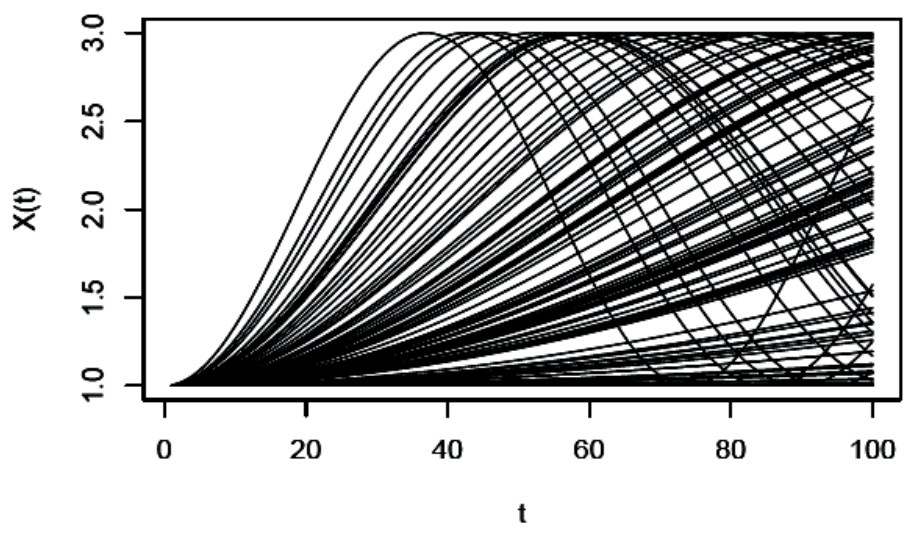

Figure 1: A sample of 100 curves $\left\{X_{i}(t), t \in[0,1]\right\}_{i=1, \ldots, 100}$

On the other side, a nonlinear functional regression, defined as follows, is considered

$$
R(X)=\frac{1}{4} \int_{0}^{1}\left(X^{\prime}(t)\right)^{2} d t
$$

the computation of our estimator is based on the observed data $\left(X_{i}, \delta_{i}, Y_{i}\right)_{i=1}^{n}$, where $Y_{i}=\min \left(T_{i}, C_{i}\right), \delta_{i}=\mathbf{1}_{\left\{T_{i} \leq C_{i}\right\}}$.

To assess the accuracy of the proposed estimator, we split the generated data into a training $(\mathcal{L})$ and a testing $(\mathcal{J})$ subsamples. The training subsample is used to estimate the single functional index and to select the smoothing parameters $h_{k}$ and $h_{H}$. Whereas the testing subsample is used to assess and compare the single functional index based estimator of the conditional quantile, namely $\widehat{\zeta}_{\theta}(\gamma, \cdot)$, to the kernel-type conditional quantile estimator, say $\widehat{\zeta}(\gamma, \cdot)$, which is introduced in Chaouch and Khardani [8] as follows:

$$
\widehat{\zeta}(\gamma, x)=\inf \left\{y \in \mathbb{R}, \widehat{F}^{x}(y) \geq \gamma\right\}
$$


where

$$
\widehat{\mathrm{F}}^{x}(y)=\frac{\sum_{i=1}^{n} \frac{\delta_{i}}{\mathrm{G}_{n}\left(Y_{i}\right)} \mathrm{K}\left(\mathrm{h}_{\mathrm{K}}^{-1} \mathrm{~d}\left(x, X_{i}\right)\right) \mathrm{H}\left(\mathrm{h}_{\mathrm{H}}^{-1}\left(y-Y_{i}\right)\right)}{\sum_{i=1}^{n} \mathrm{~K}\left(\mathrm{~h}^{-1} \mathrm{~d}\left(x, X_{i}\right)\right)}, \quad \forall y \in \mathbb{R} .
$$

Figure 2 displays the first three eigenfunctions calculated from the estimated covariance operator using the data in the training subsample.

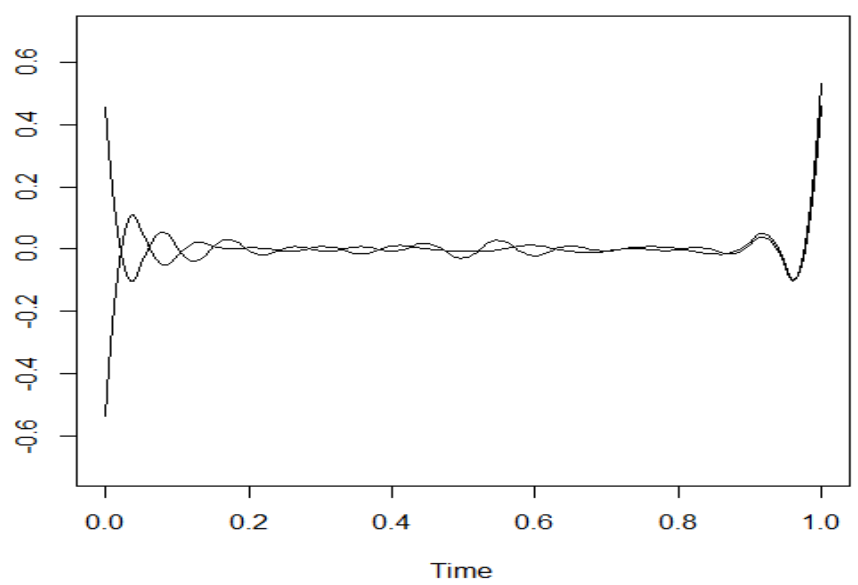

Figure 2: The first three eigenfunctions (respectively, continuous, dashed and dotted lines) representing $\theta_{i}(t), i=1,2$

Given an $X=x$, we can observe that the random variable $T$ has a normal distribution with mean equal to $R(x)$ and standard deviation equal to 0.2. Therefore, the conditional median is equal to $R(x)$. A 500 Monte-Carlo simulations are performed in order to assess the estimation accuracy of $R(x)$ using the conditional median estimation by the single functional index approach and by the nonparametric approach. The simulations were performed for two sample sizes $n=100,500$ and for two Censorship Rates $C R=60 \%, 30 \%$. Furthermore, some tuning parameters have to be specified. The kernel $\mathrm{K}(\cdot)$ is chosen to be the quadratic function defined as $\mathrm{K}(\mathrm{u})=\frac{3}{2}\left(1-\mathrm{u}^{2}\right) \mathbf{1}_{[0,1]}$ and the cumulative distribution function $\mathrm{H}(\mathfrak{u})=\int_{-\infty}^{\mathfrak{u}} \frac{3}{4}\left(1-z^{2}\right) \mathbf{1}_{[-1,1]}(z) d z$. As shown in Figure 1 the covariate is a smooth process and the regression function $R(\cdot)$ is defined as the integral of the derivative of the functional random variable $X$. Consequently, according to Ferraty and Vieu [16], the appropriate choice of the semi-metric is the $\mathrm{L}_{2}$ distance between the first derivatives of the curves. In 
this section, we assume that $h:=h_{K}=h_{H}$, is selected using a cross-validation method based on the k-nearest neighbors as described in Ferraty and Vieu [16], p. 102.

We consider the absolute error (AE) as a measure of accuracy of the estimators:

$$
A E_{k, \theta}=\left|\widehat{\zeta}_{\theta}(0.5, x)-R(x)\right| \quad \text { and } \quad A E_{k}=|\widehat{\zeta}(0.5, x)-R(x)|, \quad k=1, \ldots, 500 \text {, }
$$

where $\widehat{\zeta}_{\theta}(0.5, x)$ and $\widehat{\zeta}(0.5, x)$ are, respectively, the estimators of the conditional median using the single functional index model and the nonparametric approach. Table 1 shows that the SFIM estimator performs better that the NP one in estimating $R(x)$. Higher is the sample size and lower is the censorship rate better will be the accuracy of the SFIM compared to the NP one. Moreover, even when $\mathrm{CR}=60 \%$ and $n=100$, the SFIM estimator is still performing better than the NP one.

Table 1: First, second and third quartile of the Absolute errors $\left(A E_{k, \theta}\right.$ and $A E_{k}$, $k=1, \ldots, 500$ ) obtained for $\mathrm{CR}=60 \%$ and $\mathrm{CR}=30 \%$ (between parentheses).

\begin{tabular}{l|cc|cc}
\hline & \multicolumn{2}{|c|}{$\mathrm{n}=100$} & \multicolumn{2}{c}{$\mathrm{n}=500$} \\
& $\mathrm{NP}$ & $\mathrm{SFIM}$ & $\mathrm{NP}$ & $\mathrm{SFIM}$ \\
\hline 1st quartile of AE & 0.709 & 0.69 & 0.62 & 0.53 \\
& $(0.29)$ & $(0.212)$ & $(0.136)$ & $(0.097)$ \\
\hline Median of AE & 0.955 & 0.93 & 0.95 & 0.75 \\
& $(0.557)$ & $(0.573)$ & $(0.584)$ & $(0.346)$ \\
\hline 3rd quartile of AE & 1.085 & 1.08 & 1.07 & 0.92 \\
& $(0.73)$ & $(0.76)$ & $(0.718)$ & $(0.624)$ \\
\hline
\end{tabular}

The next phase of this simulation study consists in comparing the accuracy of the SFIM and the NP approaches in terms of prediction. For this purpose a sample of 550 observations was simulated according to the previous functional regression model defined above. A subsample of size 500 is considered for training and the remaining 50 observations are used for prediction assessment. The purpose consists in predicting the response variable $Y_{i}$ in the test sample using the conditional median which is estimated either by SFIM or NP approach. An overall assessment of the predictions is performed using the median square error, where the square error (SE) is defined as follows: $S E_{j, \theta}:=\left(Y_{j}-\widehat{\zeta}_{\theta}(0.5, x)\right)$ and $S E_{j}:=\left(Y_{j}-\widehat{\zeta}(0.5, x)\right), j=1, \ldots, 50$. Two censorship rates are considered here: $C R=45 \%$ and $C R=2 \%$. 

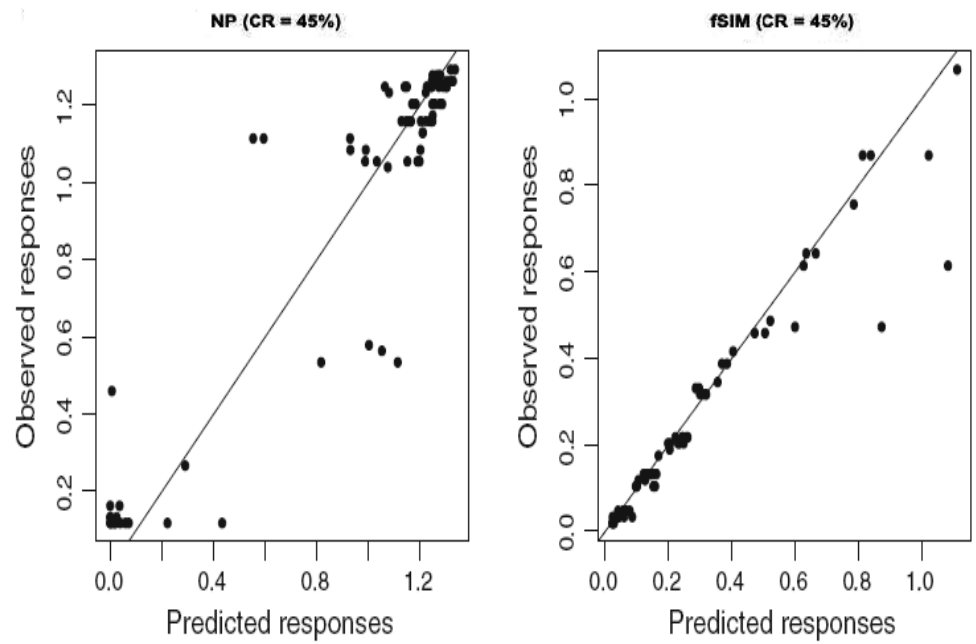

Figure 3: Prediction of $\left(Y_{j}\right)_{j=1, \ldots, 50}$ in the test subsample when $C R=45 \%$.
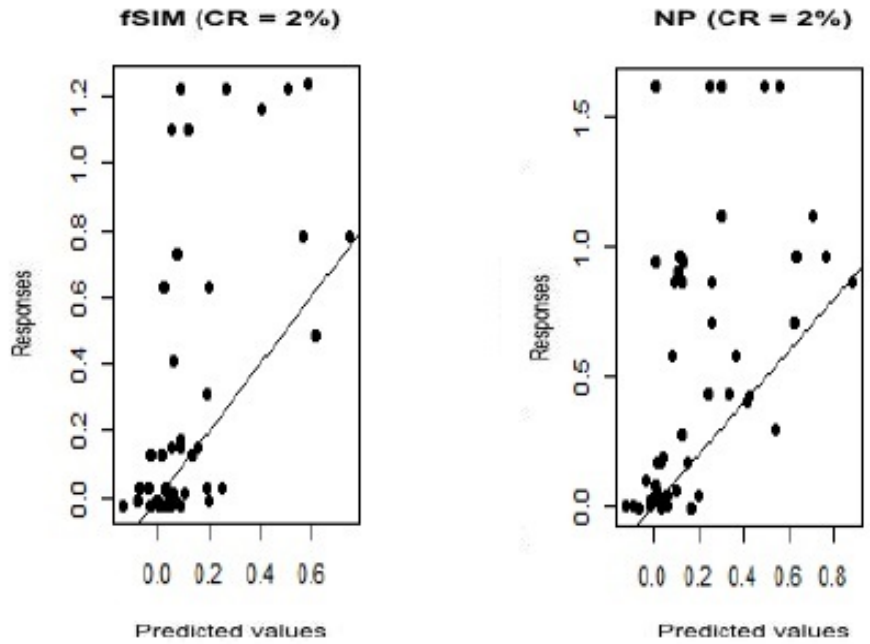

Figure 4: Prediction of $\left(Y_{j}\right)_{j=1, \ldots, 50}$ in the test subsample when $C R=2 \%$.

Figures 3 and 4 show that the SFIM estimator performs better than the NP estimator in predicting the response variable in the testing subsample. The accuracy increases when the censorship rate decreases. Indeed when $\mathrm{CR}=$ 
$45 \%$, the median square error is equal to 0.011 using the SFIM approach and 0.055 for the NP one. whereas, when $\mathrm{CR}=2 \%$, the median square error is equal to 0.008 for the SFIM and 0.012 for the NP approach.

\section{Proofs}

In order to prove our results, we introduce some further notations. Let First we consider the following decomposition

$$
\begin{aligned}
\widehat{F}(\theta, t, x)-F(\theta, t, x) & =\frac{\widehat{F}_{N}(\theta, t, x)}{\widehat{F}_{D}(\theta, x)}-\frac{a_{1}(\theta, x) F(\theta, t, x)}{a_{1}(\theta, x)} \\
& =\frac{1}{\widehat{F}_{D}(\theta, x)}\left(\widehat{F}_{N}(\theta, t, x)-\mathbb{E} \widehat{F}_{N}(\theta, t, x)\right) \\
& -\frac{1}{\widehat{F}_{D}(\theta, x)}\left(a_{1}(\theta, x) F(\theta, t, x)-\widehat{E}_{N}(\theta, t, x)\right) \\
& +\frac{F(\theta, t, x)}{\widehat{F}_{D}(\theta, x)}\left(a_{1}(\theta, x)-\mathbb{E}\left[\widehat{F}_{D}(\theta, x)\right]\right) \\
& -\frac{F(\theta, t, x)}{\widehat{F}_{D}(\theta, x)}\left(\widehat{F}_{D}(\theta, x)-\mathbb{E} \widehat{F}_{D}(\theta, x)\right) \\
& =\frac{1}{\widehat{F}_{D}(\theta, x)} A_{n}(\theta, t, x)+B_{n}(\theta, t, x)
\end{aligned}
$$

where

$$
\begin{aligned}
& A_{n}(\theta, t, x)= \frac{1}{n \mathbb{E} K_{1}(x, \theta)} \sum_{i=1}^{n}\left\{\left(\frac{\delta_{i}}{\bar{G}_{n}} H_{i}(t)-F(\theta, t, x)\right) K_{i}(\theta, x)\right. \\
&\left.\quad-\mathbb{E}\left[\left(\frac{\delta_{i}}{\bar{G}_{n}} H_{i}(y)-F(\theta, t, x)\right) K_{i}(\theta, x)\right]\right\} \\
&=\frac{1}{n \mathbb{E} K_{1}(x, \theta)} \sum_{i=1}^{n} N_{i}(\theta, t, x) .
\end{aligned}
$$

It follows that,

$$
\begin{aligned}
n \phi_{\theta, x}\left(h_{K}\right) \operatorname{Var}\left(A_{n}(\theta, t, x)\right)= & \frac{\phi_{\theta, x}\left(h_{K}\right)}{\mathbb{E}^{2} K_{1}(x, \theta)} \operatorname{Var}\left(N_{1}\right) \\
& +\frac{\phi_{\theta, x}\left(h_{K}\right)}{n \mathbb{E}^{2} K_{1}(x, \theta)} \sum_{|i-j|>0}^{n} \sum^{n} \operatorname{Cov}\left(N_{i}, N_{j}\right)
\end{aligned}
$$




$$
\begin{aligned}
= & V_{n}(\theta, t, x) \\
& +\frac{\phi_{\theta, x}\left(h_{K}\right)}{n \mathbb{E}^{2} K_{1}(x, \theta)} \sum_{|i-j|>0}^{n} \sum^{n} \operatorname{Cov}\left(N_{i}, N_{j}\right) .
\end{aligned}
$$

Lemma 1 Under hypotheses (H1)-(H3) and (H6)-(H8) as $\mathrm{n} \rightarrow \infty$ we have

$$
n \phi_{\theta, x}\left(h_{K}\right) \operatorname{Var}\left(A_{n}(\theta, t, x)\right) \longrightarrow V(\theta, t, x)
$$

where $\mathrm{V}(\theta, t, x)=\frac{a_{2}(\theta, x)}{\left(a_{1}(\theta, x)\right)^{2}} F(\theta, t, x)\left(\frac{1}{\bar{G}(t}-F(\theta, t, x)\right)$.

Lemma 2 Under hypotheses (H1)-(H3), (H6) and (H8)-(H10), as $\mathrm{n} \rightarrow \infty$ we have

$$
\left(\frac{n \phi_{\theta, x}\left(h_{k}\right)}{V(\theta, t, x)}\right)^{1 / 2} A_{n}(\theta, t, x) \stackrel{\mathcal{D}}{\longrightarrow} \mathcal{N}(0,1)
$$

where $\stackrel{\mathcal{D}}{\longrightarrow}$ denotes the convergence in distribution.

Lemma 3 Under Assumptions (H1)-(H3) and (H6)-(H9)as $\mathrm{n} \rightarrow \infty$ we have

$$
\sqrt{n \phi_{\theta, x}\left(h_{K}\right)} B_{n}(\theta, t, x) \longrightarrow 0 \text { in Probabilty. }
$$

Next, Making use of Proposition 3.2 for $\mathrm{l}=1$ and Theorem 3.1, in Kadiri et al. [18] we get the following corollary.

Corollary 1 Under hypotheses of Lemma 3, as $\mathrm{n} \rightarrow \infty$ we have

$$
\frac{\left(\mathrm{n} \phi_{\theta, x}\left(h_{\mathrm{K}}\right)\right)^{1 / 2} \mathrm{~B}_{\mathrm{n}}(\theta, \mathrm{t}, \mathrm{x})}{\widehat{\mathrm{f}}\left(\theta, \zeta_{\theta, \mathrm{n}}^{*}(\gamma, x), x\right)} \longrightarrow 0 \text { in Probabilty. }
$$

Proof. [Proof of Theorem 1]

To prove Theorem 1, it suffices to use (12). Applying Lemmas Lemma 1 and Lemma 3, we get the result.

Proof. [Proof of Theorem 2]

For Theorem 2, making use of (12), we have

$$
\begin{aligned}
\sqrt{n \phi_{\theta, x}\left(h_{K}\right)}\left(\zeta_{\theta}(\gamma, x)-\zeta_{\theta, n}(\gamma, x)\right)= & \sqrt{n \phi_{\theta, x}\left(h_{K}\right)} \frac{F_{n}\left(\theta, \zeta_{\theta}(\gamma, x), x\right)}{F_{n}^{\prime}\left(\theta, \zeta_{\theta, n}^{*}(\gamma, x), x\right)} \\
& -\sqrt{n \phi_{\theta, x}\left(h_{K}\right)} \frac{F\left(\theta, \zeta_{\theta}(\gamma, x), x\right)}{F_{n}^{\prime}\left(\theta, \zeta_{\theta, n}^{*}(\gamma, x), x\right)}
\end{aligned}
$$




$$
\begin{gathered}
=\frac{\sqrt{n \phi_{\theta, x}\left(h_{K}\right)} A_{n}(\theta, t, x)}{F_{n}^{\prime}\left(\theta, \zeta_{\theta, n}^{*}(\gamma, x), x\right)} \\
-\frac{\sqrt{n \phi_{\theta, x}\left(h_{K}\right)} B_{n}(\theta, t, x)}{F_{n}^{\prime}\left(\theta, \zeta_{\theta, n}^{*}(\gamma, x), x\right)} .
\end{gathered}
$$

Then using Theorem 1, Corollary 1 and Lemma 3 we obtain the result.

Proof. [Proof of Lemma 1]

$$
\begin{aligned}
V_{n}(\theta, t, x) & =\frac{\phi_{\theta, x}\left(h_{k}\right)}{\mathbb{E}^{2} K_{1}(\theta, x)} \mathbb{E}\left[K_{1}^{2}(\theta, x)\left(\frac{\delta_{1}}{\bar{G}\left(Y_{1}\right)} H_{1}(t)-F(\theta, t, x)\right)^{2}\right] \\
& =\frac{\phi_{\theta, x}\left(h_{K}\right)}{\mathbb{E}^{2} K_{1}(\theta, x)} \mathbb{E}\left[K_{1}^{2}(\theta, x) \mathbb{E}\left(\left(\frac{\delta_{1} H_{1}(t)}{\bar{G}\left(Y_{1}\right)}-F(\theta, t, x)\right)^{2} \mid<\theta, X_{1}>\right)\right] .
\end{aligned}
$$

Using the definition of conditional variance, we have

$$
\mathbb{E}\left[\left(\frac{\delta_{1}}{\bar{G}\left(Y_{1}\right)} \mathrm{H}\left(h_{H}^{-1}\left(t-Y_{1}\right)\right)-F(\theta, t, x)\right)^{2} \mid<\theta, X_{1}>\right]=J_{1 n}+J_{2 n}
$$

where $\mathrm{J}_{1 \mathrm{n}}=\operatorname{Var}\left(\frac{\delta_{1}}{\mathrm{G}\left(\mathrm{Y}_{1}\right)} \mathrm{H}\left(\mathrm{h}_{\mathrm{H}}^{-1}\left(\mathrm{t}-\mathrm{Y}_{1}\right)\right) \mid<\theta, \mathrm{X}_{1}>\right)$,

$$
\mathrm{J}_{2 \mathrm{n}}=\left[\mathbb{E}\left(\frac{\delta_{1}}{\mathrm{G}\left(\mathrm{Y}_{1}\right)} \mathrm{H}\left(\mathrm{h}_{\mathrm{H}}^{-1}\left(\mathrm{t}-\mathrm{Y}_{1}\right)\right) \mid<\theta, X_{1}>\right)-\mathrm{F}(\theta, \mathrm{t}, \mathrm{x})\right]^{2}
$$

Concerning $\mathrm{J}_{1 \mathrm{n}}$,

$$
\begin{aligned}
J_{1 n}= & \mathbb{E}\left[\frac{\delta_{1}}{\overline{\mathrm{G}}^{2}\left(\mathrm{Y}_{1}\right)} \mathrm{H}^{2}\left(\frac{\mathrm{t}-\mathrm{Y}_{1}}{\mathrm{~h}_{\mathrm{H}}}\right) \mid<\theta, x>\right] \\
& -\left(\mathbb{E}\left[\frac{\delta_{1}}{\overline{\mathrm{G}}\left(\mathrm{Y}_{1}\right)} \mathrm{H}\left(\frac{\mathrm{t}-\mathrm{Y}_{1}}{\mathrm{~h}_{\mathrm{H}}}\right) \mid<\theta, \mathrm{X}_{1}>\right]\right)^{2} \\
= & \mathcal{J}_{1}+\mathcal{J}_{2} .
\end{aligned}
$$

As for $\mathcal{J}_{1}$, by the property of double conditional expectation, we get that,

$$
\begin{aligned}
\mathcal{J}_{1} & =\mathbb{E}\left\{\mathbb{E}\left[\frac{\delta_{1}}{\overline{\mathrm{G}}^{2}\left(\mathrm{Y}_{1}\right)} \mathrm{H}^{2}\left(\frac{\mathrm{t}-\mathrm{Y}_{1}}{h_{\mathrm{H}}}\right) \mid<\theta, \mathrm{X}_{1}>, \mathrm{T}_{1}\right]\right\} \\
& =\mathbb{E}\left\{\frac{\delta_{1}}{\overline{\mathrm{G}}^{2}\left(\mathrm{~T}_{1}\right)} \mathrm{H}^{2}\left(\frac{\mathrm{t}-\mathrm{T}_{1}}{\mathrm{~h}_{\mathrm{H}}}\right) \mathbb{E}\left[\mathbf{1}_{\mathrm{T}_{1} \leq \mathrm{C}_{1}} \mid \mathrm{T}_{1}\right] \mid<\theta, \mathrm{X}_{1}>\right\} \\
& =\mathbb{E}\left(\frac{1}{\overline{\mathrm{G}}\left(\mathrm{T}_{1}\right)} \mathrm{H}^{2}\left(\frac{\mathrm{t}-\mathrm{T}_{1}}{\mathrm{~h}_{\mathrm{H}}}\right) \mid<\theta, \mathrm{X}_{1}>\right)
\end{aligned}
$$




$$
\begin{aligned}
& =\int_{\mathbb{R}} \frac{1}{\bar{G}(v)} H^{2}\left(\frac{t-v}{h_{H}}\right) d F\left(\theta, v, X_{1}\right) \\
& =\int_{\mathbb{R}} \frac{1}{\bar{G}\left(t-u h_{H}\right)} H^{2}(u) d F\left(\theta, t-u h_{H}, X_{1}\right) .
\end{aligned}
$$

By the first order Taylor's expansion of the function $\overline{\mathrm{G}}^{-1}(\cdot)$ around zero, one gets

$$
\begin{aligned}
\mathcal{J}_{1}= & \int_{\mathbb{R}} \frac{1}{\overline{\mathrm{G}}(\mathrm{t})} \mathrm{H}^{2}(\mathrm{u}) \mathrm{dF}\left(\theta, \mathrm{t}-u h_{\mathrm{H}}, X_{1}\right) \\
& +\frac{h_{\mathrm{H}}^{2}}{\overline{\mathrm{G}}^{2}(\mathrm{t})} \int_{\mathbb{R}} u H(u) \overline{\mathrm{G}}^{(1)}\left(\mathrm{t}^{*}\right) \mathrm{f}\left(\theta, \mathrm{t}-\mathrm{uh} \mathrm{h}_{\mathrm{H}}, X_{1}\right) \mathrm{du}+\mathrm{o}(1)
\end{aligned}
$$

where $t^{*}$ is between $t$ and $t-u h_{H}$

Under hypothesis (H7) and using hypothesis (H3)-(ii), we get

$$
\mathcal{J}_{1}^{\prime}=\frac{h_{H}^{2}}{\bar{G}^{2}(t)} \int_{\mathbb{R}} u H^{2}(t) \bar{G}^{(1)}\left(t^{*}\right) f\left(\theta, t-u h_{H}, X_{1}\right) d u=\mathcal{O}\left(h_{H}^{2}\right) .
$$

Indeed

$$
\mathcal{J}_{1}^{\prime} \leq h_{\mathrm{H}}^{2}\left(\sup _{\mathfrak{u} \in \mathbb{R}}\left|G^{\prime}(u)\right| / \bar{G}^{2}(t)\right) \int_{\mathbb{R}} u f\left(\theta, t-u h_{H}, x\right) d u .
$$

On the other hand, by integrating by part and under assumption (H3)-(i), we have

$$
\begin{aligned}
\int_{\mathbb{R}} \frac{H^{2}(u)}{\bar{G}(t)} d F\left(\theta, t-u h_{H}, X_{1}\right)= & \frac{1}{\bar{G}(t)} \int_{\mathbb{R}} 2 H(u) H^{\prime}(u) F\left(\theta, t-u h_{H}, X_{1}\right) d u \\
& -\frac{1}{\bar{G}(t)} \int_{\mathbb{R}} 2 H(u) H^{\prime}(u) F(\theta, t, x) d u \\
& +\frac{1}{\bar{G}(t)} \int_{\mathbb{R}} 2 H(u) H^{\prime}(u) F(\theta, t, x) d u .
\end{aligned}
$$

Clearly we have

$$
\int_{\mathbb{R}} 2 H(u) H^{\prime}(u) F(\theta, t, x) d u=\left[H^{2}(u) F(\theta, t, x)\right]_{-\infty}^{+\infty}=F(\theta, t, x)
$$

thus

$$
\int_{\mathbb{R}} \frac{1}{\bar{G}(t)} H^{2}(u) d F\left(\theta, t-u h_{H}, X_{1}\right)=\frac{F(\theta, t, x)}{\bar{G}(t)}+\mathcal{O}\left(h_{\mathrm{K}}^{\beta_{1}}+h_{H}^{\beta_{2}}\right) .
$$


As for $\mathrm{J}_{2 \mathrm{n}}$, by (H2), (H4) and (H5), and using Lemma 3.2 in Kadiri et al. [18] we obtain that

$$
\mathrm{J}_{2 \mathrm{n}} \longrightarrow 0, \text { as } \mathrm{n} \rightarrow \infty \text {. }
$$

- Concerning $\mathcal{J}_{2}$

$$
\begin{aligned}
\mathcal{J}_{2}^{\prime} & =\mathbb{E}\left[\frac{\delta_{1}}{\bar{G}\left(\mathrm{Y}_{1}\right)} H_{1}(t) \mid<\theta, X_{1}>\right] \\
& =\mathbb{E}\left(\mathbb{E}\left[\frac{\delta_{1}}{\bar{G}\left(\mathrm{Y}_{1}\right)} H_{1}(t) \mid<\theta, X_{1}>, T_{1}\right]\right) \\
& =\mathbb{E}\left(\frac{1}{\bar{G}\left(T_{1}\right)} H\left(\frac{t-T_{1}}{h_{H}}\right) \mathbb{E}\left[\mathbf{1}_{T_{1} \leq C_{1}} \mid T_{1}\right] \mid<\theta, X_{1}>\right) \\
& =\mathbb{E}\left(H\left(\frac{t-T_{1}}{h_{H}}\right) \mid<\theta, X_{1}>\right) \\
& =\int H\left(\frac{t-v}{h_{H}}\right) f\left(\theta, t, X_{1}\right) d v .
\end{aligned}
$$

Moreover, we have by integration by parts and changing variables

$$
\mathcal{J}_{2}^{\prime}=F(\theta, t, x) \int H^{\prime}(u) d u+\int H^{\prime}(u)\left(F\left(\theta, t-u h_{H}, x\right)-F(\theta, t, x)\right) d u
$$

the last equality is due to the fact that $\mathrm{H}^{\prime}$ is a probability density.

Thus we have:

$$
\mathcal{J}_{2}^{\prime}=\mathrm{F}(\theta, \mathrm{t}, x)+\mathcal{O}\left(h_{\mathrm{K}}^{\beta_{1}}+h_{\mathrm{H}}^{\beta_{2}}\right) .
$$

Finally by hypothesis (H5) we get $\mathcal{J}_{2} \underset{n \rightarrow \infty}{\longrightarrow} \mathrm{F}^{2}(\theta, t, x)$.

Meanwhile, by (H1), (H4), (H6) and (H8), it follows that:

$$
\frac{\phi_{\theta, x}\left(h_{K}\right) \mathbb{E} K_{1}^{2}(\theta, x)}{\mathbb{E}^{2} K_{1}(\theta, x)} \underset{n \rightarrow \infty}{\longrightarrow} \frac{a_{2}(\theta, x)}{\left(a_{1}(\theta, x)\right)^{2}} .
$$

which leads to combining equations (14)-(18)

$$
V_{n}(\theta, t, x) \underset{n \rightarrow \infty}{\longrightarrow} \frac{a_{2}(\theta, x)}{\left(a_{1}(\theta, x)\right)^{2}} F(\theta, t, x)\left(\frac{1}{\bar{G}(t)}-F(\theta, t, x)\right) .
$$

Secondly, by the boundness of $H$ and conditioning on $\left(<\theta, X_{i}>,<\theta, X_{j}>\right)$, we have

$$
\mathbb{E}\left(\left|N_{i} N_{j}\right|\right)=\mathbb{E}\left[\left(\Omega_{i}\right)\left(\Omega_{j}\right) K_{i}(\theta, x) K_{j}(\theta, x)\right]
$$




$$
\begin{aligned}
& =\mathbb{E}\left(\mathbb{E}\left[\left(\Omega_{i}\right)\left(\Omega_{j}\right) \mid<\theta, X_{i}>,<\theta, X_{j}>\right] K_{i}(\theta, x) K_{j}(\theta, x)\right) \\
& \leq\left(1+\frac{1}{\bar{G}\left(\tau_{F}\right)}\right)^{2} \mathbb{E}\left(K_{i}(\theta, x) K_{j}(\theta, x)\right) \\
& \leq C P\left(\left(X_{i}, X_{j}\right) \in B_{\theta}(x, h) \times B_{\theta}(x, h)\right) \\
& \leq C\left(\left(\frac{\phi_{\theta, x}\left(h_{K}\right)}{n}\right)^{1 / a} \phi_{\theta, x}\left(h_{K}\right)\right)
\end{aligned}
$$

where $\Omega_{i}=\frac{\delta_{i}}{\bar{G}_{i}} H_{i}(t)-F(\theta, t, x)$.

Then, taking

$$
\begin{aligned}
\frac{\phi_{\theta, x}\left(h_{K}\right)}{n \mathbb{E}^{2} K_{1}(x, \theta)} \sum_{|i-j|>0}^{n} \sum_{n} \operatorname{Cov}\left(N_{i}, N_{j}\right)= & \frac{\phi_{\theta, x}\left(h_{K}\right)}{n \mathbb{E}^{2} K_{1}(x, \theta)} \sum_{0<|i-j| \leq m_{n}}^{n} \operatorname{Cov}\left(N_{i}, N_{j}\right) \\
& +\frac{\phi_{\theta, x}\left(h_{K}\right)}{n \mathbb{E}^{2} K_{1}(x, \theta)} \sum_{|i-j|>m_{n}}^{n} \operatorname{Cov}\left(N_{i}, N_{j}\right) \\
= & K_{1 n}+K_{2 n} .
\end{aligned}
$$

Therefore

$$
\mathrm{K}_{1 \mathrm{n}} \leq \mathrm{C} \mathrm{m}_{\mathrm{n}}\left\{\left(\frac{\phi_{\theta, \mathrm{x}}\left(\mathrm{h}_{\mathrm{K}}\right)}{\mathrm{n}}\right)^{1 / \mathrm{a}}\right\}, \quad \forall i \neq j .
$$

Now choose $m_{n}=\left(\frac{\phi_{\theta, x}\left(h_{K}\right)}{n}\right)^{-1 / a}$, we get $K_{1 n}=o(1)$.

For $K_{2 n}$ : since the variable $\left(\Delta_{i}\right)_{1 \leq i \leq n}$ is bounded (i.e, $\left\|\Delta_{i}\right\|_{\infty}<\infty$, we can use the Davydov-Rio's inequality. So, we have for all $i \neq j$,

$$
\left|\operatorname{Cov}\left(\Delta_{i}, \Delta_{j}\right)\right| \leq \operatorname{C} \alpha(|i-j|) .
$$

By the fact, $\sum_{k \geq m_{n}+1} k^{-a} \leq \int_{m_{n}}^{\infty} v^{-a} d v=\frac{m_{n}^{-a+1}}{a-1}$, we get by applying (H1),

$$
K_{2 n} \leq \sum_{|i-j| \geq m_{n}+1}|i-j|^{-a} \leq \frac{n m_{n}^{-a+1}}{a-1}
$$

with the same choice of $m_{n}$, we get $K_{2 n}=o(1)$. 
Finally by

$$
\frac{\phi_{\theta, x}\left(h_{K}\right)}{n \mathbb{E}^{2} K_{1}(x, \theta)} \sum_{|i-j|>0}^{n} \sum^{n} \operatorname{Cov}\left(N_{i}, N_{j}\right)=o(1),
$$

this complete the proof of lemma.

Proof. [Proof of Lemma 2]

We will establish the asymptotic normality of $A_{n}(\theta, t, x)$ suitably normalized. We have

$$
\begin{aligned}
\sqrt{n \phi_{\theta, x}\left(h_{K}\right)} A_{n}(\theta, t, x) & =\frac{\sqrt{n \phi_{\theta, x}\left(h_{K}\right)}}{n \mathbb{E} K_{1}(\theta, x)} \sum_{i=1}^{n} N_{i}(\theta, t, x) \\
& =\frac{\sqrt{\phi_{\theta, x}\left(h_{K}\right)}}{\sqrt{n} \mathbb{E} K_{1}(\theta, x)} \sum_{i=1}^{n} N_{i}(\theta, t, x) \\
& =\frac{1}{\sqrt{n}} \sum_{i=1}^{n} \Xi_{i}(\theta, t, x)=\frac{1}{\sqrt{n}} S_{n} .
\end{aligned}
$$

Now we can write, $\Xi_{i}=\frac{\sqrt{\phi_{\theta, x}\left(h_{K}\right)}}{\mathbb{E} K_{1}(\theta, x)} N_{i}$, we have

$$
\operatorname{Var}\left(\Xi_{i}\right)=\frac{\phi_{\theta, x}\left(h_{K}\right)}{\mathbb{E}^{2} K_{1}(\theta, x)} \operatorname{Var}\left(N_{i}\right)=V_{n}(\theta, t, x)
$$

Note that by (19), we have $\operatorname{Var}\left(\Xi_{i}\right) \longrightarrow V(\theta, t, x)$ as $n$ goes to infinity and by $(20)$, we have

$$
\sum_{|i-j|>0}\left|\operatorname{Cov}\left(\Xi_{i}, \Xi_{j}\right)\right|=\frac{\phi_{\theta, x}\left(h_{K}\right)}{\mathbb{E}^{2} K_{1}(x, \theta)} \sum_{|i-j|>0}^{n}\left|\operatorname{Cov}\left(N_{i}, N_{j}\right)\right|=o(n),
$$

Obviously, we have

$$
\sqrt{\frac{n \phi_{\theta, x}\left(h_{K}\right)}{V(\theta, t, x)}}\left(A_{n}(\theta, t, x)\right)=(n V(\theta, t, x))^{-1 / 2} S_{n} .
$$

Thus, the asymptotic normality of $(n V(\theta, t, x))^{-1 / 2} S_{n}$, is sufficient to show the proof of this Lemma. This last is shown by the blocking method, where the random variables $\Xi_{i}$ are grouped into blocks of different sizes defined. 
We consider the classical big- and small-block decomposition. We split the set $\{1,2, \ldots, n\}$ into $2 k_{n}+1$ subsets with large blocks of size $u_{n}$ and small blocks of size $v_{n}$ and put

$$
k_{n}:=\left[\frac{n}{u_{n}+v_{n}}\right] .
$$

Now by Assumption (H10)-(ii) allows us to define the large block size by

$$
u_{n}=:\left[\left(\frac{n \phi_{\theta, x}\left(h_{K}\right)}{q_{n}}\right)^{1 / 2}\right] .
$$

Using Assumption (H10) and simple algebra allows us to prove that

$$
\frac{v_{n}}{u_{n}} \rightarrow 0, \quad \frac{u_{n}}{n} \rightarrow 0, \quad \frac{u_{n}}{\sqrt{n \phi_{\theta, x}\left(h_{K}\right)}} \rightarrow 0, \quad \text { and } \quad \frac{n}{u_{n}} \alpha\left(v_{n}\right) \rightarrow 0 .
$$

Now, let $\Upsilon_{j}, \Upsilon_{j}^{\prime}$ and $\Upsilon_{j}^{\prime \prime}$ be defined as follows:

$$
\begin{aligned}
& \Upsilon_{j}(\theta, t, x)=\Upsilon_{j}=\sum_{i=j(u+v)+1}^{j(u+v)+u} \Xi_{i}(\theta, t, x), \quad 0 \leq j \leq k-1, \\
& \Upsilon_{j}^{\prime}(\theta, t, x)=\Upsilon_{j}^{\prime}=\sum_{i=j(u+v)+u+1}^{(j+1)(u+v)} \Xi_{i}(\theta, t, x), \quad 0 \leq j \leq k-1, \\
& \Upsilon_{j}^{\prime \prime}(\theta, t, x)=\Upsilon_{j}^{\prime \prime}=\sum_{i=k(u+v)+1}^{n} \Xi_{i}(\theta, t, x), \quad 0 \leq j \leq k-1 .
\end{aligned}
$$

Clearly, we can write

$$
\begin{aligned}
S_{n}(\theta, t, x)=S_{n} & =\sum_{j=1}^{k-1} \Upsilon_{j}+\sum_{j=1}^{k-1} \Upsilon_{j}^{\prime}+\Upsilon_{k}^{\prime \prime} \\
=: & \Psi_{n}(\theta, t, x)+\Psi_{n}^{\prime}(\theta, t, x)+\Psi_{n}^{\prime \prime}(\theta, t, x) \\
=: & \Psi_{n}+\Psi_{n}^{\prime}+\Psi_{n}^{\prime \prime} .
\end{aligned}
$$

We prove that
(i) $\frac{1}{n} \mathbb{E}\left(\Psi_{n}^{\prime}\right)^{2} \longrightarrow 0$,
(ii) $\frac{1}{n} \mathbb{E}\left(\Psi_{n}^{\prime \prime}\right)^{2} \longrightarrow 0$, 


$$
\begin{gathered}
\left|\mathbb{E}\left\{\exp \left(i z n^{-1 / 2} \Psi_{n}\right)\right\}-\prod_{j=0}^{k-1} \mathbb{E}\left\{\exp \left(i z n^{-1 / 2} \Upsilon_{j}\right)\right\}\right| \longrightarrow 0 \\
\frac{1}{n} \sum_{j=0}^{k-1} \mathbb{E}\left(\Upsilon_{j}^{2}\right) \longrightarrow V(\theta, t, x) \\
\frac{1}{n} \sum_{j=0}^{k-1} \mathbb{E}\left(\Upsilon_{j}^{2} \mathbf{1}_{\left\{\left|\Upsilon_{j}\right|>\varepsilon \sqrt{n V(\theta, t, x)\}}\right.}\right) \longrightarrow 0
\end{gathered}
$$

for every $\varepsilon>0$.

Expression (23) show that the terms $\Psi_{n}^{\prime}$ and $\Psi_{n}^{\prime \prime}$ are asymptotically negligible, while Equations (24) and (25) show that the $\Upsilon_{j}$ are asymptotically independent, verifying that the sum of their variances tends to $V(\theta, t, x)$. Expression (26) is the Lindeberg-Feller's condition for a sum of independent terms. Asymptotic normality of $S_{n}$ is a consequence of Equations (23)-(26).

- Proof of (23) Because $\mathbb{E}\left(\Xi_{j}\right)=0, \forall j$, we have that

$$
\mathbb{E}\left(\Psi_{n}^{\prime}\right)^{2}=\operatorname{Var}\left(\sum_{j=1}^{k-1} \Upsilon_{j}^{\prime}\right)=\sum_{j=1}^{k-1} \operatorname{Var}\left(\Upsilon_{j}^{\prime}\right)+\sum_{|i-j|>0}^{k-1} \operatorname{Cov}\left(\Upsilon_{i}^{\prime}, \Upsilon_{j}^{\prime}\right):=\Pi_{1}+\Pi_{2} .
$$

By the second-order stationarity and (21) we get

$$
\begin{aligned}
\operatorname{Var}\left(\Upsilon_{j}^{\prime}\right) & =\operatorname{Var}\left(\sum_{i=j\left(u_{n}+v_{n}\right)+u_{n}+1}^{(j+1)\left(u_{n}+v_{n}\right)} \Xi_{i}(\theta, t, x)\right) \\
& =v_{n} \operatorname{Var}\left(\Xi_{1}(x)\right)+\sum_{|i-j|>0}^{v_{n}} \operatorname{Cov}\left(\Xi_{i}(\theta, t, x), \Xi_{j}(\theta, t, x)\right) \\
& =v_{n} \operatorname{Var}\left(\Xi_{1}(x)\right)+o\left(v_{n}\right) .
\end{aligned}
$$

Then

$$
\begin{aligned}
\frac{\Pi_{1}}{\mathrm{n}} & =\frac{\mathrm{k} v_{\mathrm{n}}}{\mathrm{n}} \operatorname{Var}\left(\Xi_{1}(\theta, \mathrm{t}, \mathrm{x})\right)+\frac{\mathrm{k}}{\mathrm{n}} \mathrm{o}\left(v_{\mathrm{n}}\right) \\
& \leq \frac{\mathrm{k} v_{\mathrm{n}}}{\mathrm{n}}\left\{\frac{\phi_{\theta, x}\left(\mathrm{~h}_{\mathrm{K}}\right)}{\mathbb{E}^{2} \mathrm{~K}_{1}(\mathrm{x})} \operatorname{Var}\left(\Xi_{1}(\mathrm{x})\right)\right\}+\frac{\mathrm{k}}{\mathrm{n}} \mathrm{o}\left(v_{\mathrm{n}}\right)
\end{aligned}
$$




$$
\leq \frac{k v_{n}}{n}\left\{\frac{1}{\phi_{\theta, x}\left(h_{k}\right)} \operatorname{Var}\left(\Xi_{1}(x)\right)\right\}+\frac{k}{n} o\left(v_{n}\right)
$$

Simple algebra gives us

$$
\frac{k v_{n}}{n} \cong\left(\frac{n}{u_{n}+v_{n}}\right) \frac{v_{n}}{n} \cong \frac{v_{n}}{u_{n}+v_{n}} \cong \frac{v_{n}}{u_{n}} \longrightarrow 0 \quad \text { as } n \rightarrow \infty .
$$

Using Equation (20) we have

$$
\lim _{n \rightarrow \infty} \frac{\Pi_{1}}{n}=0
$$

Now, let us turn to $\Pi_{2} / \mathrm{n}$. We have

$$
\begin{aligned}
\frac{\Pi_{2}}{n} & =\frac{1}{n} \sum_{|i-j|>0}^{k-1} \operatorname{Cov}\left(\Upsilon_{i}(x), \Upsilon_{j}(x)\right) \\
& =\frac{1}{n} \sum_{|i-j|>0}^{k-1} \sum_{l_{1}=1}^{v_{n}} \sum_{l_{2}=1}^{v_{n}} \operatorname{Cov}\left(\Xi_{m_{j}+l_{1}}, \Xi_{m_{j}+l_{2}}\right),
\end{aligned}
$$

with $m_{i}=i\left(u_{n}+v_{n}\right)+u_{n}+1$. As $i \neq j$, we have $\left|m_{i}-m_{j}+l_{1}-l_{2}\right| \geq u_{n}$. It follows that

$$
\frac{\Pi_{2}}{n} \leq \frac{1}{n} \sum_{|i-j| \geq u_{n}}^{n} \operatorname{Cov}\left(\Xi_{i}(x), \Xi_{j}(x)\right)=o(1),
$$

then

$$
\lim _{n \rightarrow \infty} \frac{\Pi_{2}}{n}=0
$$

By Equations (27) and (28) we get Part(i) of the Equation(23).

We turn to (ii), we have

$$
\begin{aligned}
\frac{1}{n} \mathbb{E}\left(\Psi_{n}^{\prime \prime}\right)^{2} & =\frac{1}{n} \operatorname{Var}\left(\Upsilon_{k}^{\prime \prime}\right) \\
& =\frac{\vartheta_{n}}{n} \operatorname{Var}\left(\Xi_{1}(x)\right)+\frac{1}{n} \sum_{|i-j|>0}^{\vartheta_{n}} \operatorname{Cov}\left(\Xi_{i}(x), \Xi_{j}(x)\right),
\end{aligned}
$$

where $\vartheta_{n}=n-k_{n}\left(u_{n}+v_{n}\right)$; by the definition of $k_{n}$, we have $\vartheta_{n} \leq u_{n}+v_{n}$. 
Then

$$
\frac{1}{n} \mathbb{E}\left(\Psi_{n}^{\prime \prime}\right)^{2} \leq \frac{u_{n}+v_{n}}{n} \operatorname{Var}\left(\Xi_{1}(x)\right)+\frac{1}{n} \sum_{|i-j|>0}^{\vartheta_{n}} \operatorname{Cov}\left(\Xi_{i}(x), \Xi_{j}(x)\right)
$$

and by the definition of $\boldsymbol{u}_{n}$ and $v_{n}$ we achieve the proof of (ii) of Equation $(23)$.

- Proof of (24) We make use of Volkonskii and Rozanov's lemma (see the appendix in Masry [26]) and the fact that the process $\left(\mathbf{X}_{\boldsymbol{i}}, \mathbf{X}_{\mathbf{j}}\right)$ is strong mixing.

Note that $\Upsilon_{a}$ is $\mathcal{F}_{i_{a}}^{j_{a}}$-mesurable with $i_{a}=a\left(u_{n}+v_{n}\right)+1$ and $j_{a}=$ $a\left(u_{n}+v_{n}\right)+u_{n}$; hence, with $v_{j}=\exp \left(i z n^{-1 / 2} \Psi_{n}\right)$ we have

$$
\begin{aligned}
\left|\mathbb{E}\left\{\mathrm{V}_{\mathrm{j}}\right\}-\prod_{j=0}^{k-1} \mathbb{E}\left\{\exp \left(i z n^{-1 / 2} \Upsilon_{j}\right)\right\}\right| & \leq 16 k_{n} \alpha\left(v_{n}+1\right) \\
& \cong \frac{n}{v_{n}} \alpha\left(v_{n}+1\right)
\end{aligned}
$$

which goes to zero by the last part of Equation (22). Now we establish Equation (25).

- Proof of (25) Note that $\operatorname{Var}\left(\Psi_{n}\right) \longrightarrow V(\theta, t, x)$ by equation (23) (by the definition of the $\Xi_{i}$ ). Then because

$$
\mathbb{E}\left(\Psi_{n}\right)^{2}=\operatorname{Var}\left(\Psi_{n}\right)=\sum_{j=0}^{k-1} \operatorname{Var}\left(\Upsilon_{j}\right)+\sum_{i=0}^{k-1} \sum_{i \neq j}^{k-1} \operatorname{Cov}\left(\Upsilon_{i}, \Upsilon_{j}\right)
$$

all we have to prove is that the double sum of covariances in the last equation tends to zero. Using the same arguments as those previously used for $\Pi_{2}$ in the proof of first term of Equation (23) we obtain by replacing $v_{n}$ by $u_{n}$ we get

$$
\frac{1}{n} \sum_{j=1}^{k-1} \mathbb{E}\left(\Upsilon_{j}^{2}\right)=\frac{k u_{n}}{n} \operatorname{Var}\left(\Xi_{1}\right)+o(1)
$$

As $\operatorname{Var}\left(\Xi_{1}\right) \longrightarrow \mathrm{V}(\theta, \mathrm{t}, x)$ and $\frac{\mathrm{k} u_{n}}{n} \longrightarrow 1$, we get the result.

Finally, we prove Equation (26). 
- Proof of (26) Recall that

$$
\Upsilon_{j}=\sum_{i=j\left(u_{n}+v_{n}\right)+1}^{j\left(u_{n}+v_{n}\right)+u_{n}} \Xi_{i} .
$$

Finally for establish (26) it suffices to show for $n$ large enough that the set $\left\{\left|\Upsilon_{j}\right|>\varepsilon \sqrt{n V(\theta, t, x)}\right\}$ is empty .

Making use Assumptions (H3) and (H5), we have

$$
\left|\Xi_{i}\right| \leq C\left(\phi_{\theta, x}\left(h_{K}\right)\right)^{-1 / 2}
$$

therefore

$$
\left|\Upsilon_{j}\right| \leq C u_{n}\left(\phi_{\theta, x}\left(h_{k}\right)\right)^{-1 / 2}
$$

which goes to zero as $\mathfrak{n}$ goes to infinity by Equation (22).

Since $\left|H_{i}(t)-F(\theta, t, x)\right| \leq 1$, then

$$
\begin{aligned}
\left|\Upsilon_{j}\right| & \leq \frac{u_{n} N_{j}}{\sqrt{\phi_{\theta, x}\left(h_{K}\right)}} \\
& \leq \frac{C u_{n}}{\sqrt{\phi_{\theta, x}\left(h_{K}\right)}}
\end{aligned}
$$

Thus

$$
\frac{1}{\sqrt{n}}\left|\Upsilon_{j}\right| \leq \frac{C u_{n}}{\sqrt{n \phi_{\theta, x}\left(h_{K}\right)}}
$$

Then for $n$ large enough, the set $\left\{\left|\Upsilon_{j}\right|>\varepsilon(n V(\theta, t, x))^{-1 / 2}\right\}$ becomes empty, this completes the proof and therefore that of the asymptotic normality of $(\mathrm{nV}(\theta, \mathrm{t}, \mathrm{x}))^{-1 / 2} \mathrm{~S}_{\mathrm{n}}$ and the Lemma 2.

\section{Proof. [Proof of Lemma 3]}

We have

$$
\begin{array}{r}
\sqrt{n \phi_{\theta, x}\left(h_{K}\right)} B_{n}(\theta, t, x)=\frac{\sqrt{n \phi_{\theta, x}\left(h_{K}\right)}}{\widehat{F}_{D}(\theta, x)}\left\{\mathbb{E} \widehat{F}_{N}(\theta, t, x)-a_{1}(\theta, x) F(\theta, t, x)\right. \\
\left.+F(\theta, t, x)\left(a_{1}(\theta, x)-\mathbb{E} \widehat{F}_{D}(\theta, x)\right)\right\} .
\end{array}
$$


Firstly, observed that the results below as $n \rightarrow \infty$

$$
\begin{gathered}
\frac{1}{\phi_{\theta, x}\left(h_{K}\right)} \mathbb{E}\left[K^{l}\left(\frac{<x-X_{i}, \theta>}{h_{K}}\right)\right] \longrightarrow a_{l}(\theta, x), \text { for } l=1,2, \\
\mathbb{E}\left[\widehat{F}_{D}(\theta, x)\right] \longrightarrow a_{1}(\theta, x),
\end{gathered}
$$

and

$$
\mathbb{E}\left[\widehat{\mathrm{F}}_{\mathrm{N}}(\theta, t, x)\right] \longrightarrow \mathrm{a}_{1}(\theta, x) \mathrm{F}(\theta, t, x),
$$

can be proved in the same way as in Ezzahrioui and Ould-Saïd [14] corresponding to their Lemmas 5.1 and 5.2, and then their proofs are omitted.

Secondly, on the one hand, making use of (29), (30) and (31), we have as $n \rightarrow \infty$

$$
\left\{\mathbb{E} \widehat{F}_{N}(\theta, t, x)-a_{1}(\theta, x) F(\theta, t, x)+F(\theta, t, x)\left(a_{1}(\theta, x)-\mathbb{E} \widehat{F}_{D}(\theta, x)\right)\right\} \longrightarrow 0 .
$$

On other hand,

$$
\frac{\sqrt{n \phi_{\theta, x}\left(h_{K}\right)}}{\widehat{F}_{D}(\theta, x)}=\frac{\sqrt{n \phi_{\theta, x}\left(h_{K}\right)} \widetilde{F}^{\prime}(\theta, t, x)}{\widehat{F}_{D}(\theta, x) \widetilde{F}^{\prime}(\theta, t, x)}=\frac{\sqrt{n \phi_{\theta, x}\left(h_{K}\right)} \widetilde{F}^{\prime}(\theta, t, x)}{\widetilde{F}_{N}^{\prime}(\theta, t, x)} .
$$

Then using Proposition 3.2 in Kadiri et al. [18], it suffices to show that $\frac{\sqrt{n \phi_{\theta, x}\left(h_{k}\right)}}{\widetilde{F}_{N}^{\prime}(\theta, t, x)}$ tends to zero as $n$ goes to infinity.

Indeed

$$
\widetilde{\mathrm{F}}_{\mathrm{N}}^{\prime}(\theta, \mathrm{t}, x)=\frac{1}{n \mathrm{~h}_{\mathrm{H}} \mathbb{E} \mathrm{K}_{1}(\theta, x)} \sum_{i=1}^{n} \frac{\delta_{i}}{\overline{\mathrm{G}}\left(\mathrm{Y}_{\mathrm{i}}\right)} \mathrm{K}\left(\frac{<x-X_{i}, \theta>}{h_{\mathrm{K}}}\right) \mathrm{H}^{\prime}\left(\frac{\mathrm{t}-\mathrm{Y}_{\mathrm{i}}}{\mathrm{h}_{\mathrm{H}}}\right) .
$$

Because $\mathrm{K}(\cdot) \mathrm{H}^{\prime}(\cdot)$ is continuous with support on $[0,1]$ then by $(\mathrm{H} 5)$-(ii) and (H6) $\exists \mathrm{m}=\inf _{[0,1]} \mathrm{K}(\mathrm{t}) \mathrm{H}^{\prime}(\mathrm{t})$ it follows that

$$
\widetilde{\mathrm{F}}_{\mathrm{N}}^{\prime}(\theta, \mathrm{t}, x) \geq \frac{\mathrm{m}}{\mathrm{h}_{\mathrm{H}} \phi_{\theta, \mathrm{x}}\left(\mathrm{h}_{\mathrm{K}}\right)}
$$

which gives

$$
\frac{n \phi_{\theta, x}\left(h_{K}\right)}{\widetilde{F}_{N}^{\prime}(\theta, t, x)} \leq \frac{\sqrt{n h_{H}^{2} \phi_{\theta, x}\left(h_{K}\right)^{3}}}{m} .
$$

Finally, using (H10), completes the proof of Lemma 3. 


\section{References}

[1] A. Aït Saidi, F. Ferraty and R. Kassa, Single functional index model for a time series, Revue Roumaine de Mathématique Pures Appliquées, 50 (2002), 321-330.

[2] A. Aït Saidi, F. Ferraty, R. Kassa and P. Vieu, Cross-validated estimation in the single functional index model, Statistics, 42(6) (2008), 475-494.

[3] S. Attaouti, A. Laksaci and E. Ould-Saïd, A note on the conditional density estimate in the single functional index model, Statistics and Probability Letters, 81(1) (2011), 45-53.

[4] S. Attaoui, On the Nonparametric Conditional Density and Mode Estimates in the Single Functional Index Model with Strongly Mixing Data, Sankhyã: The Indian Journal of Statistics, 76(A) (2014), 356-378.

[5] S. Attaoui and N. Ling, Asymptotic Results of a Nonparametric Conditional Cumulative Distribution Estimator in the single functional index Modeling for Time Series Data with Applications, Metrika, 79 (2016), $485-511$.

[6] Z. Cai, Estimating a distribution function for censored time series data, J. Multivariate Anal., 78 (2001), 299-318.

[7] Z. Cai, Regression quantiles for time series, Econometric Theory, 18 (2002), 169-192.

[8] M. Chaouch and S. Khardani, Randomly Censored Quantile Regression Estimation using Functional Stationary Ergodic Data, Journal of Nonparametric Statistics, 27 (2015), 65-87.

[9] P. Chaudhuri, K. Doksum and A. Samarov, On average derivative quantile regression, Ann. Statist., 25 (1997),715-744.

[10] J. Dedecker, P. Doukhan, G. Lang, J.R. Leon, S. Louhichi and C. Prieur, Weak Dependence: With Examples and Applications, Lecture Notes in Statistics, 190. New York: Springer-Verlag, 2007.

[11] P. Doukhan, Mixing: Properties and Examples, Lecture Notes in Statistics, 85. New York: Springer-Verlag, 1994.

[12] J. L. Doob, Stochastic Processes, New York: Wiley, 1953. 
[13] A. El Ghouch and I. Van Keilegom, Local Linear Quantile Regression with Dependent Censored Data, Statistica Sinica, 19 (2009), 1621-1640.

[14] M. Ezzahrioui and E. Ould-Saïd, Asymptotic results of a nonparametric conditional quantile estimator for functional time series, Comm. Statist. Theory and Methods., 37(16-17) (2008), 2735-2759.

[15] F. Ferraty, A. Rabhi and P. Vieu, Conditional quantiles for functional dependent data with application to the climatic ElNinõ phenomenon, Sankhyã B, Special Issue on Quantile Regression and Related Methods, 67(2) (2005), 378-399.

[16] F. Ferraty and P. Vieu, Nonparametric Functional Data Analysis: Theory and Practice, Springer Series in Statistics, Springer, New York, 2006.

[17] A. Gannoun, J. Saracco and K. Yu, Nonparametric prediction by conditional median and quantiles, J. Statist. Plann. Inference., 117 (2003), $207-223$.

[18] N. Kadiri, A. Rabhi and A. Bouchentouf, Strong uniform consistency rates of conditional quantile estimation in the single functional index model under random censorship, Journal Dependence Modeling, 6(1) (2018), $197-227$.

[19] E. Kaplan and P. Meier, Nonparametric Estimation from Incomplete Observations, Journal of the American Statistical Association, 53 (1958), $457-481$.

[20] J-P. Lecoutre and E. Ould-Saïd, Hazard rate estimation for strong mixing and censored processes, J. Nonparametr. Stat. 5 (1995), 83-89.

[21] H.Y. Liang and J. Ua-lvarez, Asymptotic Properties of Conditional Quantile Estimator for Cen- sored Dependent Observations, Annals of the Institute of Statistical Mathematics, 63 (2011), 267-289.

[22] Z. Lin and C. Lu, Limit theory of mixing dependent random variables, Mathematics and its applications, Sciences Press, Kluwer Academic Publishers, Beijing, 1996.

[23] N. Ling and Q. Xu, Asymptotic normality of conditional density estimation in the single index model for functional time series data, Statistics and Probability Letters, 82 (2012), 2235-2243. 
[24] N. Ling, Z. Li and W. Yang, Conditional density estimation in the single functional index model for $\alpha$-mixing functional data, Communications in Statistics: Theory and Methods, 43(3) (2014), 441-454.

[25] E. Masry and D. Tøjstheim, Nonparametric estimation and identification of nonlinear time series, Econometric Theor., 11 (1995), 258-289.

[26] E. Masry, Nonparametric Regression Estimation for Dependent Functional Data: Asymptotic Normality, Stochastic Processes and their Applications, 115 (2005), 155-177.

[27] C. Muharisa, F. Yanuar and D. Devianto, Simulation Study The Using of Bayesian Quantile Regression in Non-normal Error, Cauchy: Jurnal Matematika Murni dan Aplikasi, 5(3) (2018), 121-126.

[28] E. Ould-Saï, A Strong Uniform Convergence Rate of Kernel Conditional Quantile Estima- tor under Random Censorship, Statistics and Probability Letters, 76 (2006), 579-586.

[29] W-J. Padgett, Nonparametric Estimation Of Density And Hazard Rate Functions When Samples Are Censored, In P.R. Krishnaiah and C.R. Rao (Eds). Handbook of Statist. 7, pp. 313-331. Elsevier/North-Holland. Amsterdam. Science Publishers, 1988.

[30] G. Roussas, Nonparametric estimation of the transition distribution function of a Markov process, Ann. Statist., 40 (1969), 1386-1400.

[31] M. Samanta, Nonparametric estimation of conditional quantiles, Statist. Probab. Lett., 7 (1989), 407-412.

[32] S. Sarmada and F. Yanuar, Quantile Regression Approach to Model Censored Data, Science and Technology Indonesia, 5(3) (2020), 79-84.

[33] M. Tanner and W-H. Wong, The estimation of the hazard function from randomly censored data by the kernel methods, Ann. Statist., 11 (1983), 989-993.

[34] I. Van-Keilegom and N. Veraverbeke, Hazard rate estimation in nonparametric regression with censored data, Ann. Inst. Statist. Math., 53 (2001), $730-745$.

[35] H. Wang and Y. Zhao, A kernel estimator for conditional t-quantiles for mixing samples and its strong uniform convergence, (in chinese), Math. Appl. (Wuhan)., 12 (1999), 123-127. 
[36] F. Yanuar, H. Laila and D. Devianto, The Simulation Study to Test the Performance of Quantile Regression Method With Heteroscedastic Error Variance, Cauchy: Jurnal Matematika Murni dan Aplikasi, 5(1) (2017), $36-41$.

[37] F. Yanuar, H. Yozza, F. Firdawati, I. Rahmi, and A. Zetra, Applying Bootstrap Quantile Regression for The Construction of a Low Birth Weight Model, Makara Journal of Health Research, 23(2) (2019), 90-95.

[38] Y. Zhou and H. Liang, Asymptotic properties for $\mathrm{L}_{1}$ norm kernel estimator of conditional median under dependence, J. Nonparametr. Stat., 15 (2003), 205-219.

Received: August 31, 2020 